\title{
Validation of a Measure of Alliance for an Adolescent Inpatient Setting
}

\author{
Greg Haggerty, ${ }^{1 *}$ Caleb J. Siefert, ${ }^{2}$ Samuel J. Sinclair, ${ }^{3}{ }^{3}$ ennifer Zodan, ${ }^{1}$ \\ Ronke Babalola ${ }^{1}$ and Mark A. Blais ${ }^{3}$ \\ ${ }^{1}$ Von Tauber Institute for Global Psychiatry, Nassau University Medical Center, East Meadow, NY, USA \\ ${ }^{2}$ University of Michigan-Dearborn, Dearborn, MI, USA \\ ${ }^{3}$ Massachusetts General Hospital, Harvard Medical School, Boston, MA, USA
}

\begin{abstract}
The link between alliance and treatment outcome is robust. Nevertheless, few, if any, self-report measures exist to assess the alliance between hospitalized adolescents and their treatment team as a whole. The present study looks to extend the use of a brief self-report measure of inpatient treatment alliance designed for adult inpatients to be used with adolescents. The scale is designed incorporating items that tap the three factors of alliance (bond, goals and collaboration) to assess the alliance that the patient has with his or her treatment team. Our results show that the Inpatient-Treatment Alliance Scale is unifactoral, shows good psychometrics and is linked in theoretically meaningful ways to global clinician ratings of engagement in individual psychotherapy. Copyright $\odot 2014$ John Wiley \& Sons, Ltd.

Key Practitioner Message:

- Inpatient treatment of adolescents requires the assessment of alliance to be between the patient and his or her treatment team rather than an individual clinician.

- Assessment of the alliance can benefit clinicians treating hospitalized adolescents especially because these patients are difficult to engage with in treatment.

- This study shows that the Inpatient-Treatment Alliance Scale is a promising measure for assessing treatment alliance on an adolescent inpatient setting.
\end{abstract}

Keywords: Adolescents, Alliance, Inpatient, I-TAS, SOS-10

Therapeutic alliance is one of the most extensively researched topics in treatment research (Horvath, Del Re, Fluckiger, \& Symonds, 2011). The term commonly refers to an agreement between patient and therapist on the tasks and goals of therapy and the collaborative bond (Bordin, 1979). In a recent meta-analysis by Horvath et al. (2011), it showed that alliance was found to account for $7.5 \%$ of the variance in outcome in adult treatment. Research findings show that therapeutic alliance plays a significant role in all psychiatric outpatient treatment that includes psychotherapy as well as psychopharmacology (Blatt \& Zuroff, 2005; Fuertes et al., 2014; Hilsenroth \& Cromer, 2007; Krupnick et al., 1996).

Even though evidence is strong for the relationship between alliance and treatment outcome for outpatient treatment, relatively little research exists investigating the relationship with inpatient populations. Inpatient alliance research has lagged probably for reasons related to the complexity in assessing it in these settings. Alliance

*Correspondence to: Greg Haggerty, Von Tauber Institute for Global Psychiatry/Nassau University Medical Center, 320 1st Avenue, Massapequa Park, NY 11762, USA.

E-mail: Ghag541@aol.com research with outpatient samples investigates the relationship between two people, the patient and therapist. In contrast, inpatient treatment incorporates alliances with multiple providers (i.e., psychiatrist, psychologist, social worker, nurses and recreation therapists) and in a variety of settings (individual and group therapies; Blais, 2004). A decreasing length of stay on inpatient services has also added to the difficulty in assessing inpatient alliance (News \& Notes, 2003). Hospitalized patients also demonstrate complex and more severe psychopathology than those seen for outpatient care.

Research investigating inpatient therapeutic alliance reveals its importance in effective care. Blais, Jacobo and Smith (2010) used a patient and therapist version of a six-item six-point scale assessing inpatient psychotherapy alliance based on Hatcher and Barends (1996) research assessing four primary alliance factors: treatment goals, bond, collaboration and affective tone. They found that both patient and therapist ratings of alliance were linked with ratings of global functioning and patient perceived depth of psychotherapy. Clarkin, Hurt and Crilly (1987) used a single-item, six-point clinician-rated scale that equated alliance with treatment engagement and found that alliance was positively related to ratings of functioning at 
discharge and independently predicted outcome. Allen et al. (1986) also used a single-item, six-point staff rating scale to assess the degree to which patients collaborated with their treatment. Greater collaboration was deemed a marker of better alliance. Allen et al. (1986) found that these ratings were significantly related to ratings of global functioning both at the early stages of treatment and at discharge. Lieberman, von Rehn, Dickie, Elliott and Egerter (1992) used greater agreement between patient and staff on treatment goals and expected benefits of treatment as a marker of better alliance. They found that greater agreement at admission was linked with decreases in symptomatology and a lower risk of patients leaving against medical advice or being transferred.

Relatively few studies have investigated alliance with adolescents on an inpatient service. Kroll and Green (1997) addressed the measurement issue by developing and validating a measure of inpatient engagement with adolescent inpatients. The measure they created, Inpatient Engagement Questionnaire, is a 16-item clinician-rated form. In this study, a nurse who was familiar with the patient would complete the questionnaire a month after admission. The shortcoming with this measure is that it is clinician rated, and research has shown that observer-rated alliance ratings are only weakly related to outcome (McLeod, 2011). The study also suffered from a small sample size of 30 patients. In another study Hintikka, Laukkanen, Marttunen and Lehtonen (2006) looked at how alliance was related to positive cognitive changes in adolescent inpatients. The study showed that alliance was positive correlated with cognitive performance changes at discharge. This study does not address the patient's alliance with the treatment team at large.

In this paper, we looked to extend the use of an inpatient alliance measure, the Inpatient-Treatment Alliance Scale (I-TAS; Blais, 2004) that was originally designed for use with adults to adolescent inpatients. The I-TAS may help address gaps in the inpatient alliance literature. The I-TAS is a patient-rated questionnaire in the Appendix that assesses therapeutic alliance between the patient and his or her treatment team. It is also a brief, easy to administer and score measure that could facilitate its use on busy inpatient units. We used the same version that has been used with adults (Blais, 2004). Having a similar measure for both adult and adolescent inpatients allows researchers the ability to investigate developmental differences between the two groups. We present preliminary psychometric data and construct validity data for the I-TAS with an adolescent inpatient sample.

The I-TAS (Blais, 2004) was created to assess the patient's composite treatment alliance as it develops across the total inpatient hospitalization. A review of the factor analysis of Hatcher and Barends (1996) of three widely used alliance measures (i.e., Working Alliance Inventory, California Psychotherapy Alliance Questionnaire and the Penn Helping Alliance Questionnaire) guided the item selection for the I-TAS. The I-TAS was developed to tap the primary alliance factors identified by Hatcher and Barends (1996) of bond (I-TAS items 4, 6 and 8), goals (items 2, 5, 7 and 9) and collaboration (items 1, 3 and 10). Item wording was modified to better match the inpatient alliance experience. The I-TAS was limited to 10 items to lessen patient burden. Blais (2004) had 140 adult inpatients complete the I-TAS at admission, between the fourth and sixth day of hospitalization and at discharge. Results revealed that the I-TAS was unifactoral with one primary factor having an eigenvalue of 6.6 and accounting for $66.4 \%$ of the total variance. The factor loadings for all 10 I-TAS items were from 0.63 to 0.90 . Internal consistency was found to be $\alpha=0.91$. Early treatment I-TAS scores were related to patient satisfaction, length of hospital stay and patients' initial reduction in symptom severity (change in symptoms assessed at admission and again between the fourth and sixth day of hospitalization).

In this paper, we investigated the factor structure and internal consistency of the I-TAS with adolescent inpatients. We use the same version of the I-TAS that was previously used with adults (Blais, 2004). We hypothesized that the I-TAS would demonstrate a similar factor structure to Blais (2004) in that it would be unifactoral and show good internal consistency. We also investigated the construct validity of the I-TAS by correlating it to the clinician-rated global engagement ratings for individual and group therapy. We hypothesized that the I-TAS would be positively correlated with clinician-rated engagement ratings of individual and group therapy.

\section{METHODS}

\section{Participants}

The sample consisted of 72 patients $(45 \%$ of the total patients admitted to the unit), 52.8\% male patients, consecutively admitted to the Adolescent Psychiatric Inpatient Unit of a large north-eastern hospital. This study was approved by the hospital's Institutional Review Board of record. Patients were between the ages of 13-17 years old with a mean age of 15.7 years old (standard deviation [SD] = 1.18). Ethnic makeup of the sample was as follows: $40.8 \%$ Caucasian, 25.4\% African American, 25.4\% Latino/ Hispanic/Spanish, 5.6\% others and 2.8\% Asian. The primary diagnosis for these 72 patients was as follows: $64 \%$ mood disorders, 30\% conduct disorder/oppositional defiant disorder, $2 \%$ impulse control disorder, $3 \%$ psychosis and $1 \%$ post traumatic stress disorder. We found no significant differences in age, gender, diagnosis and scores on admission measure between those who consented to the study versus those who did not consent.

\section{Setting}

The adolescent inpatient unit in this study is a 12-bed locked facility at a large north-eastern hospital. The unit 
provides treatment for adolescents in acute distress. The average length of stay for patients on this unit is 10.81 days $(\mathrm{SD}=5.23)$. While on the unit, adolescents receive psychopharmalogical treatment as deemed necessary, two to three individual psychotherapy sessions per week, three general group sessions per week, a weekly structured anger management group, a weekly substance use psychoeducation group, daily academic programming and daily recreation therapy.

Individual therapy on the unit is structured around assisting in alleviating the patient's acute symptomatology so as to stabilize the patient. Therapy is provided by either a licensed psychologist with over 5 years of experience or an advanced psychology graduate trainee (psychology extern or psychology intern as part of the hospital's American Psychological Association [APA]-accredited internship) who is supervised by a licensed psychologist. The therapy provided is integrative in nature. Therapists meet with adolescents individually and work together to identify goals for therapy in the first session. A mixture of cognitive behavioural and insight-oriented interventions is then used to help clients work towards these goals. Adolescents are encouraged to discuss feelings about their struggles, interpersonal patterns and then work with therapists to identify methods for working towards goals while on the unit. In addition, therapists and adolescents focus on interactions with other members of the unit staff and other patients. In the final session with the adolescents, therapists assess how the client feels he or she is doing, explore the client's experience of the hospital, discuss how gains for inpatient stay can be continued and review how the client feels about discharge. On average, participants received three individual therapy sessions $(\mathrm{SD}=1.56)$. These numbers are based on actual sessions attended.

Group therapy sessions on the unit were delivered by members of the psychology staff. All groups are co-led by the licensed unit psychologist, psychology extern or psychology intern. Group work by externs and interns is supervised by a licensed unit psychologist. Groups are offered five times per week on the unit, and adolescents were encouraged to attend all groups offered. All group therapy sessions are open groups; thus, participants in the group may change daily. Three groups are semi-structured, free speech groups in which members were free to talk about various topics affecting them. The fourth group is focused on anger management and is informed by dialectical behaviour therapy. A fifth group is focused on educating the patients about the consequences of substance, understanding the potential triggers for use and available outpatient resources. On average, participants engaged in six group sessions $(\mathrm{SD}=3.08)$ during their stay. These numbers are based on actual group therapy sessions attended.

All patients, whether they were enrolled in this study or not, received individual psychotherapy and group therapy as part of their treatment on the unit. Patients on the unit were assigned to an individual and group psychotherapist in an ecologically valid manner based on clinician availability and caseload. The patient's individual therapist completed the global assessment of the patient's engagement in individual therapy, and the group therapist did the same assessment for group therapy. The individual and group therapists were blind to each others' ratings both for admission and discharge measures.

\section{Study Therapists/Raters}

Participating clinicians included one licensed clinical psychologist with over 5 years of experience post-graduate and advanced training in assessment and five (four female students) advanced clinical psychology doctoral students (three interns and two externs) who had completed advanced coursework in assessment at an APA-accredited clinical psychology Ph.D. program and were supervised by a licensed clinical psychologist. The trainees' ages ranged from 27 to 35 years old with an average age of 29.3 years old. Two of the three psychology interns had extensive training in the therapeutic model of assessment (Finn \& Tonsager, 1997) and the study measures before their participation in this study. The psychology interns had 4.3 years of training, and both psychology externs had 3 years of training before starting their rotation on the unit. All clinicians kept a caseload of two patients throughout their rotation on the unit. This caseload was not limited to consented patients. At any given time, the unit contained a licensed psychologist, a psychology intern and an extern. All study clinicians met on a bi-weekly basis for reliability training for clinical rating measure.

\section{Measures}

\section{Inpatient-Treatment Alliance Scale}

Inpatient-Treatment Alliance Scale (Blais, 2004) is a 10-item Likert-style self-report measure designed to assess the patient's composite treatment alliance and engagement as it developed across the entire inpatient treatment experience. I-TAS items were selected through a guided review of the factor analysis by Hatcher and Barends (1996) of three widely used alliances measures developed for outpatient individual treatment. In developing the I-TAS, 10 items were selected that cover the alliance factors of bond (items 4, 6 and 8), goals (items 2, 5, 7 and 9) and collaboration (items 1, 3 and 10). The items were worded to better match the inpatient treatment experience. The scale was limited to 10 items to reduce patient burden. The authors reviewed the items and felt they were not inappropriate for adolescents to answer and were worded in an age appropriate manner. Blais (2004) found the mean score for each item was 4.55 (1.35) with adult inpatients. 


\section{Clinician Therapy Engagement Rating}

The patients' individual and group psychotherapist rated the overall amount of engagement and participation that the patient showed in the modality that they treated them (i.e., group or individual therapy) during the length of their hospitalization. They rated them on a scale from 1 ('not engaged') to 5 ('very engaged'). Therapists were instructed to rate patients as engaged in the therapeutic activity if they were actively initiating conversations and, in group, also providing constructive feedback to other group members. The therapist also had to feel as if the patient took responsibility for his or her problems and was motivated to improve. A rating of ' 1 ' was indicative of a patient who did not speak or pay attention in therapy and/or refused to attend therapeutic activities. A rating of ' 5 ' was indicative of a patient who took responsibility for his or her behavioural problems, showed that they put thought between sessions to what he/she was working on with his/her therapist and in group provided constructive feedback. This rating was performed blind to the other clinicians' ratings of each patient as well as self-report questionnaire results. The rating was also completed at the patient's discharge. Raters were instructed to rate the patients overall averaged level of engagement. Training for this included reviewing patients known to the raters but not part of the study to provide guidance on scoring this measure. Raters had to evidence at least an intraclass correlation coefficient of 0.70 during the training sessions for this measure.

\section{Procedure}

Parents and legal guardians were approached for participation by the research team member who was not one of the clinicians in the study at the end of the initial family meeting. All patients who were diagnosed as cognitively impaired (IQ 70) were excluded from study participation. At or within 1 day of discharge, patients completed a discharge assessment packet that included the I-TAS (Blais, 2004). Patients were told that their therapists would not be made aware of their self-reported data both in writing and also verbally by the research assistant before they completed the assessments. The patient's individual group therapist completed a global rating of engagement in individual psychotherapy, while the patient's group therapist completed a global rating of engagement in individual psychotherapy. Both individual and group therapists were blind to each others' ratings as well as to the patients' I-TAS results.

\section{RESULTS}

Means and standard deviations for the study measures are reported in Table 1 . To evaluate the factor structure of the I-TAS, an exploratory principal component analysis
Table 1. Means and standard deviations for the I-TAS and the global ratings of engagement in individual and group therapy

\begin{tabular}{lccc}
\hline & $n$ & Mean & SD \\
\hline I-TAS & 60 & 4.67 & 1.16 \\
$\begin{array}{l}\text { Global rating of engagement } \\
\text { in individual therapy }\end{array}$ & 57 & 3.28 & 1.19 \\
$\begin{array}{l}\text { Global rating of engagement } \\
\text { in group therapy }\end{array}$ & 65 & 2.71 & 1.11 \\
Number of medications $^{\dagger}$ & 60 & 1.39 & .83 \\
\hline
\end{tabular}

Note: I-TAS = Inpatient Treatment Alliance Scale.

${ }^{+}=$number of medications the patient was on at the time that they completed the discharge measures that included the I-TAS.

was employed. This analysis yielded two components with eigenvalues greater than 1. Parallel analysis (PA; Horn, 1965) and scree plot review were used to determine the number of factors to be retained (Figure 1). The PA is an empirically supported component retention procedure that involves creating a series of random data matrices with the same number of variables (i.e., columns) and participants (i.e., rows) as the study correlation matrix. PA then generates eigenvalues for the random matrices for comparison against the genuine (actual) eigenvalues. For a component to be retained, the actual eigenvalue should be larger than the 95th percentile of the corresponding average randomly generated eigenvalue. In the present study, the actual eigenvalues for the first two components were 5.8 and 1.06, respectively, while the first two PA eigenvalues were 1.58 and 1.41, respectively. Applying PA retention guidelines suggests that only a single component should be extracted. For a detailed description of the PA procedure refer to Hoelzle and Meyer (2009). All 10 items of the I-TAS had factor loadings greater than 0.68 . Table 2 lists the means, standard deviations and ceiling and flooring effects of each item of the I-TAS as well as the adjusted item-scale correlations. The adjusted

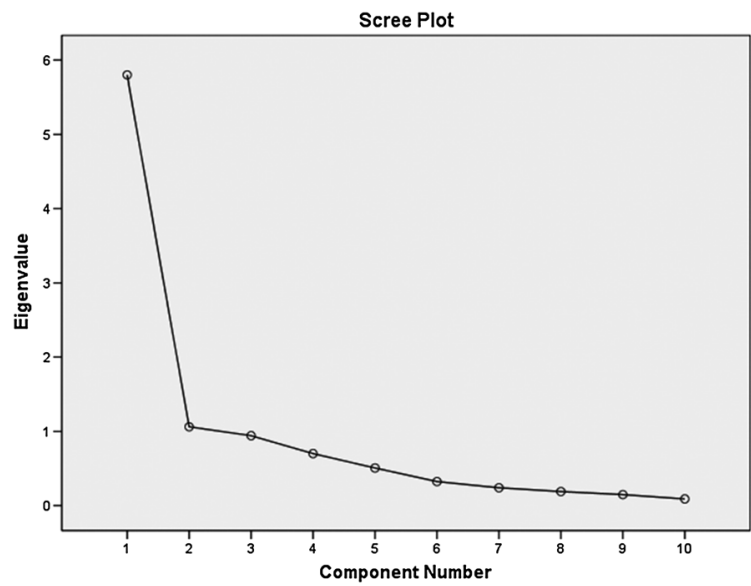

Figure 1. Scree plot of I-TAS principal component analysis 
Table 2. Means and standard deviation of I-TAS, ceiling effects, flooring effects and adjusted item-scale correlations

\begin{tabular}{lccccc}
\hline $\begin{array}{l}\text { I-TAS } \\
\text { Item }\end{array}$ & Mean & $\begin{array}{c}\text { Standard } \\
\text { deviation }\end{array}$ & $\begin{array}{c}\text { Ceiling } \\
\text { effects }\end{array}$ & $\begin{array}{c}\text { Flooring } \\
\text { effects }\end{array}$ & $\begin{array}{c}\text { Adjusted item-scale } \\
\text { correlation }\end{array}$ \\
\hline 1 & 4.64 & 1.35 & $1.6 \%$ & $31.1 \%$ & 0.70 \\
2 & 4.39 & 1.73 & $6.6 \%$ & $29.5 \%$ & 0.69 \\
3 & 4.57 & 1.49 & $0 \%$ & $34.4 \%$ & 0.65 \\
4 & 4.52 & 1.51 & $1.6 \%$ & $32.8 \%$ & 0.78 \\
5 & 4.84 & 1.54 & $1.6 \%$ & $42.6 \%$ & 0.65 \\
6 & 4.93 & 1.35 & $3.3 \%$ & $45.9 \%$ & 0.68 \\
7 & 4.21 & 1.65 & $4.9 \%$ & $23 \%$ & 0.80 \\
8 & 4.67 & 1.70 & $4.9 \%$ & $39.3 \%$ & 0.73 \\
9 & 4.79 & 1.58 & $3.3 \%$ & $47.5 \%$ & 0.61 \\
10 & 4.85 & 1.53 & $4.9 \%$ & $47.5 \%$ & 0.68 \\
\hline
\end{tabular}

Note: Cronbach alpha $=0.92$; ceiling effects refer to the percentage of participants who gave a 6 for that item (highest score possible); flooring effects refer to the percentage of participants who gave a 0 for that item (lowest score possible.

item-to-scale correlations were also higher than 0.61. These findings are in line with the research on the I-TAS in adult inpatient samples (Blais, 2004). Table 3 also lists the rotated and unrotated factor loadings for the two factors that had eigenvalues above 1 .

We performed Pearson product-moment correlations to investigate the construct validity of the I-TAS. Table 4 contains the correlational results between the I-TAS and the global ratings of engagement in individual and group therapy. I-TAS scores were significantly positively related to global ratings of engagement in individual psychotherapy but not for group therapy.

\section{DISCUSSION}

The concept of therapeutic alliance is nothing to new to child and adolescent treatment. Unfortunately, research

Table 3. Principal component analysis with Promax rotation with Kaiser normalization for the I-TAS

\begin{tabular}{lrrc}
\hline Item & Factor $1^{\dagger}$ & Factor $^{+}$ & Unrotated factor 1 \\
\hline I-TAS 1 & $\mathbf{0 . 8 8}$ & -0.06 & 0.76 \\
I-TAS 2 & $\mathbf{0 . 7 5}$ & 0.08 & 0.76 \\
I-TAS 3 & 0.05 & $\mathbf{0 . 7 5}$ & 0.72 \\
I-TAS 4 & $\mathbf{0 . 7 1}$ & 0.20 & 0.83 \\
I-TAS 5 & $\mathbf{1 . 0 1}$ & -0.23 & 0.73 \\
I-TAS 6 & 0.15 & $\mathbf{0 . 6 9}$ & 0.74 \\
I-TAS 7 & 0.49 & 0.46 & 0.85 \\
I-TAS 8 & $\mathbf{0 . 5 3}$ & 0.35 & 0.79 \\
I-TAS 9 & -0.29 & $\mathbf{1 . 0 7}$ & 0.68 \\
I-TAS 10 & 0.20 & $\mathbf{0 . 6 3}$ & 0.74 \\
\hline
\end{tabular}

Note: $n=60$.

${ }^{+}=$Factors from pattern matrix; unambiguous loadings (i.e., a factor loading $>0.40$ and at least $0.10>$ than loading on other factor) are shown in bold type. Unrotated factor 1 presents the factor loading for the first unrotated principle axis factor.
Table 4. Pearson product correlations between I-TAS and global rating of engagement in individual psychotherapy

\begin{tabular}{lrrrr}
\hline & & \multicolumn{2}{c}{ I-TAS } \\
\cline { 4 - 5 } & $n$ & $R$ & $P$ \\
\hline Global rating of engagement in individual & 49 & 0.39 & 0.006 \\
psychotherapy & & & \\
Global rating of engagement in group therapy & 55 & 0.02 & 0.87 \\
Number of individual therapy sessions & 51 & 0.09 & 0.52 \\
Number of group therapy session & 55 & -0.03 & 0.86 \\
Length of stay in days & 54 & -0.01 & 0.96 \\
Number of psychotropic medications ${ }^{\dagger}$ & 60 & 0.07 & 0.61 \\
\hline${ }^{\dagger}=$ The number of psychotropic medications the patient was on at the time \\
they completed the I-TAS.
\end{tabular}

on the topic is far behind that of adult alliance research. Research on patient's alliance with the inpatient treatment teams is even scarcer. This is unfortunate considering the prohibitive cost of hospitalizing children and adolescents and the lack of inpatient hospital beds. The problem is twofold. Existing alliance measures were created to assess alliance in individual treatment and not with a treatment team. Second, the measures that exist are either clinician or observer rated (which has historically shown a weaker link with outcome than self-reported alliance) or have too many items to be utilized on fast-paced (often scarcely staffed) inpatients settings.

Our results reveal that the I-TAS is unifactoral. This result is in line with what has been found when the I-TAS has been used with adult inpatient samples (Blais, 2004) and illustrates that all I-TAS items are tapping one common latent variable. Results also reveal support of the use of the single total score of the I-TAS for research. This finding raises the possibility that the structure of alliance on an inpatient setting may differ from outpatient settings.

Results also support the construct validity of the I-TAS as a measure of inpatient treatment alliance with adolescents. Our results are compelling because scores on the I-TAS were compared with clinician-rated measures that were blind to the patient's I-TAS scores. Our results reveal that I-TAS scores were linked with the individual therapists' global rating of engagement in individual psychotherapy. We would expect that if someone has a good alliance that they would engage more in their therapy. Future research still needs to identify which one leads to the other. Patients may report better alliances after being more engaged in their treatment. We were surprised that I-TAS scores were not related to the global engagement for group therapy rating. We have no way to look further into the reasons for this in our dataset. Several moderating factors could be the patient's shyness, interpersonal style and management of perception by others. Patients could be engaged in their overall treatment but be cautious in a group therapy setting with other patients who have conduct problems and antisocial tendencies. 
We compared the mean I-TAS score from Blais' (2004) adult inpatient sample and our adolescent inpatient sample and found that our sample had a slightly higher mean item score. Our analysis revealed that the difference between the samples were not statistically different $(t=0.60$, degrees of freedom $=198, p=0.55$.). This is somewhat surprising given that the average age in each sample was so different ( 47 years old versus 15.7 years old). Future research would be needed to better understand this lack of difference. The mean score on the I-TAS of 4.67 tells us that most patients felt that their treatment was listening to their concerns and collaborating with them. Overall, they felt their hospitalization was a success. If we look at the item level, we see that the two lowest scored items (items 2 and 7) show that inpatient clinicians could engage their patients more actively in a treatment planning process by listening more intently to their concerns.

Every study has limitations and this one is no exception. The I-TAS was given at discharge, and perhaps future research should administer the I-TAS 2 or 3 days after admission and also a couple of days before discharge or at least before the patient is aware that they are being discharge. We decided to administer the I-TAS at discharge in this study because we felt patients would be more truthful about their perceptions of their treatment team when they knew that the answers would not affect their discharge. Even though patients were explicitly made aware that their answers would not be disclosed to the treatment team staff, adolescent patients who are often hospitalized against their will may not always be the most trusting of adults. Our study also did not include any validity measures. We have no way of knowing how honestly the patients completed the study measures. Our results are what would be expected given what we know about alliance and the I-TAS. The item average and standard deviations were in line with results from Blais (2004). Future research should utilize validity measures as a way to control for the integrity of the data. Another limitation is that our measure of treatment engagement was a one-item measure rated by the therapist. Future research would do well to use a different measure of treatment engagement.

One of the strengths of our study is that we compared questionnaire results from the patients themselves to clinician-rated measures. We also used the measure in a clinical setting with a patient population with which is difficult to engage. We feel that the results demonstrate the I-TAS's promise as a measure of adolescent inpatient alliance. It is quick and easy to administer and score, which is important for measures being used on a fastpaced inpatient service. This not only lessens patient fatigue but also clinician fatigue in completing study measures. The I-TAS showed good psychometrics and was linked in theoretically important ways with the other study measures.

\section{ACKNOWLEDGEMENTS}

This study was funded in part by a grant awarded to the first author from the American Psychoanalytic Association and by the National Institute of Mental Health Grant No. 1R21MH097781-01A1 received by the first author.

\section{REFERENCES}

Allen, J. G., Tarnoff, G., Coyne, L., Spohn, H. E., Buskirk, J. R., \& Keller, M. W. (1986). An innovative approach to assessing outcome of long-term psychiatric hospitalization. Psychiatric Services, 37(4), 376-380.

Blais, M. A. (2004). Development of an inpatient treatment alliance scale. Journal of Nervous and Mental Disease, 192, 487-493.

Blais, M. A., Jacobo, M. C., \& Smith, S. R. (2010). Exploring therapeutic alliance in brief inpatient psychotherapy: A preliminary study. Clinical Psychology and Psychotherapy, 17, 386-394.

Blatt, S. J., \& Zuroff, D. C. (2005). Empirical evaluation of the assumptions in identifying evidence based treatments in mental health. Clinical Psychology Review, 25(4), 459-486.

Bordin, E. S. (1979). The generalizability of the psychoanalytic concept of the working alliance. Psychotherapy: Theory, Research, and Practice, 16, 252-260.

Clarkin, J. F., Hurt, S. W., \& Crilly, J. L. (1987). Therapeutic alliance and hospital treatment outcome. Hospital and Community Psychiatry, 38, 871-875.

Finn, S. E., \& Tonsager, M. E. (1997). Information-gathering and therapeutic models of assessment: Complementary paradigms. Psychological Assessment, 9(4), 374.

Fuertes, J. N., Anand, P., Haggerty, G., Kestenbaum, M., Rosenblum, G. (2014). The Physician-Patient Working Alliance and Patient Psychological Attachment, Adherence, Outcome Expectations, and Satisfaction in a Sample of Rheumatology Patients. Behavioral Medicine. DOI:10.1080/08964289.2013.875885.

Hatcher, R. L., \& Barends, A. W. (1996). Patients' view of alliance in psychotherapy: Exploratory factor analysis of three alliance measures. Journal of Consulting and Clinical Psychology, 64, 1326-1336.

Hilsenroth, M. J., \& Cromer, T. D. (2007). Clinician interventions related to alliance during the initial interview and psychological assessment. Psychotherapy: Theory, Research, Practice, Training, 44(2), 205.

Hintikka, U., Laukkanen, E., Marttunen, M., \& Lehtonen, J. (2006). Good working alliance and psychotherapy are associated with positive change in cognitive performance among adolescent psychiatric inpatients. Bulletin of the Menninger Clinic, 70(4), 316-335.

Hoelzle, J. B., \& Meyer, G. J. (2009). The invariant component structure of the PersonalityAssessment Inventory (PAI) full scales. Journal of Personality Assessment, 91, 175-186.

Horn, J. L. (1965). A rationale and test for the number of factors in factor analysis. Psychometrika, 30, 179-185.

Horvath, A. O., Del Re, A. C., Fluckiger, C., \& Symonds, D. (2011). Alliance in individual psychotherapy. Psychotherapy, 48(1), 9-16.

Kroll, L., \& Green, J. (1997). The therapeutic alliance in child inpatient treatment: Development and initial validation of a Family Engagement Questionnaire. Clinical Child Psychology and Psychiatry, 2(3), 431-447. 
Krupnick, J. L., Sotsky, S. M., Simmens, S., Moyer, J., Elkin, I., Watkins, J., \& Pilkonis, P. A. (1996). The role of the therapeutic alliance in psychotherapy and pharmacotherapy outcome: findings in the National Institute of Mental Health Treatment of Depression Collaborative Research Program. Journal of Consulting and Clinical Psychology, 64(3), 532.

Lieberman, P. B., von Rehn, S., Dickie, E., Elliott, B., \& Egerter, E. (1992). Therapeutic effects of brief hospitalization: the role of a therapeutic alliance. The Journal of Psychotherapy Practice and Research, 1(1), 56.

McLeod, B. D. (2011). Relation of alliance with outcomes in youth psychotherapy: A meta-analysis. Clinical Psychology Review, 31, 603-616.

News \& Notes. (2003). Occupancy rates in psychiatric hospitals climbs while length of stay reach historic low, survey finds. Psychiatric Services, 54, 585-586.

\section{APPENDIX A: THE INPATIENT-TREATMENT ALLIANCE SCALE (I-TAS)}

Instructions: We are interested in hearing about how you feel your hospital treatment is going so far. We are particularly interested in knowing how well you feel you are working with your treatment team as a whole. What we mean by your treatment team is all the unit staff members who work regularly with you during your stay here. We are collecting this information as part of a quality improvement process, and your questionnaires will not be reviewed until after you leave the hospital.

Please read the statements below and circle the number that best fits how you feel about your treatment team right now.

1) I feel I am working well with my treatment team

2) I feel that my treatment team has good understanding of my problems.

3) I feel that my treatment team listens to concerns.

4) I feel that someone from my treatment team will be available if I need them.

5) I feel that my treatment team wants me to participate fully in my treatment.

6) I feel that my treatment team wants to help me.

7) I felt like an active member of my treatment team

8) I feel respected by my treatment team.

9) My treatment team and I agree about what needs to change so I can leave the hospital.

10) I feel that my hospital treatment will be successful.

\begin{tabular}{lllllcl} 
False & & \multicolumn{4}{c}{ Completely true } \\
0 & 1 & 2 & 3 & 4 & 5 & 6 \\
0 & 1 & 2 & 3 & 4 & 5 & 6 \\
0 & 1 & 2 & 3 & 4 & 5 & 6 \\
0 & 1 & 2 & 3 & 4 & 5 & 6 \\
0 & 1 & 2 & 3 & 4 & 5 & 6 \\
0 & 1 & 2 & 3 & 4 & 5 & 6 \\
0 & 1 & 2 & 3 & 4 & 5 & 6 \\
0 & 1 & 2 & 3 & 4 & 5 & 6 \\
0 & 1 & 2 & 3 & 4 & 5 & 6 \\
0 & 1 & 2 & 3 & 4 & 5 & 6
\end{tabular}

(C) 2004 The General Hospital Corporation doing business as Massachusetts General Hospital 